\title{
Relaxations semi-linéaire et cinétique des systèmes de lois de conservation
}

\author{
par \\ Denis SERRE ${ }^{1}$ \\ Unité de Mathématiques Pures et Appliquées, (CNRS UMR \#5669), Ecole Normale \\ Supérieure de Lyon, 46, Allée d'Italie, F-69364 Lyon Cedex 07, France \\ Manuscrit reçu 12 Mai 1998
}

Abstract. - R. DiPerna (1983) proved the convergence of the approximate solutions given by the vanishing viscosity method, towards an entropy solution of the underlying hyperbolic system. He used two main assumptions: the existence of convex positively invariant domains (in the sense of K.N. Chuey et al. (1977)) and genuine nonlinearity. We prove below that, under the same assumptions together with the subcharacteristic condition, the approximate solutions given by the semilinear relaxation converge too. Actually, our result stands for a more general approximation, first introduced by R. Natalini (1998).

(C) 2000 L'Association Publications de l'Institut Henri Poincaré. Published by Elsevier B.V. All rights reserved

Key words: Relaxation, invariant domains, convex entropies

AMS classification: $35 \mathrm{~L} 45,35 \mathrm{~L} 65$

RÉSUMÉ. - R. DiPerna (1983) a montré la convergence de la solution approchée fournie par la méthode de viscosité, vers une solution entropique du système hyperbolique sous-jacent. Il a utilisé deux hypothèses fondamentales : l'existence de domaines convexes positivement invariants (au sens de K.N. Chuey et al. (1977)) et la non-linéarité des champs caractéristiques. Nous montrons ici que ces hypothèses, jointes

\footnotetext{
${ }^{1}$ E-mail: serre@ umpa.ens-lyon.fr.
} 
à la condition sous-caractéristique, assurent aussi la convergence des solutions approchées fournies par la relaxation semi-linéaire. En fait, notre analyse est valide pour une classe plus générale d'approximations, introduites récemment par R. Natalini (1998).

(C) 2000 L'Association Publications de l'Institut Henri Poincaré. Published by Elsevier B.V. All rights reserved

Mots Clés: Relaxation, domaines invariants, entropies convexes

\section{INTRODUCTION}

Soit un système hyperbolique de lois de conservation, que nous appelons "système à l'équilibre" :

$$
\partial_{t} u+\partial_{x} f(u)=0, \quad x \in \mathbb{R}, t>0,
$$

muni d'une condition initiale $u_{0}$ mesurable bornée. Etant donnés deux nombres $\tau>0$ (le "temps de relaxation") et $a>0$ (une vitesse de propagation), nous considérons dans un premier temps le système semilinéaire

$$
\left\{\begin{aligned}
\partial_{t} u^{\tau}+\partial_{x} v^{\tau} & =0, \\
\partial_{t} v^{\tau}+a^{2} \partial_{x} u^{\tau} & =\tau^{-1}\left(f\left(u^{\tau}\right)-v^{\tau}\right),
\end{aligned}\right.
$$

qu'on appelle "relaxation semi-linéaire du système (1)". Dans (1), $u$ est à valeurs dans un convexe $\mathcal{U}$ de $\mathbb{R}^{n}$, d'intérieur non vide, tandis que (2) comprend deux fois plus d'équations et d'inconnues. Le champ $f$ est de classe $\mathcal{C}^{2}$, avec une différentielle $d f(u)$ diagonalisable à valeurs propres réelles en tout point. Enfin, $v_{0}(x)=v^{\tau}(x, 0)$ est donné dans $L^{\infty}\left(\mathbb{R}^{n}\right)$. On dit que la condition initiale est "préparée" si de plus $v_{0}=f \circ u_{0}$.

L'approximation par relaxation, du problème de Cauchy pour (1), a été envisagée par Whitham [24] puis considérée, dans un cadre plus large, par Chen, Liu et Levermore [12,2,3]. Cependant, la relaxation semi-linéaire, dans laquelle la partie principale est linéaire et à coefficients constants, a été introduite par Jin et Xin [10], en vue de construire des schémas numériques robustes. Le lecteur intéressé par un exposé général sur la relaxation pourra se reporter au mémoire de Natalini [15].

La question essentielle est la convergence de $u^{\tau}$ vers $u$, la solution entropique de (1), lorsque celle-ci existe. A notre connaissance, la plupart des réponses à ce jour concernent la relaxation d'une équation 
scalaire (le cas $n=1$, voir [2,14,5,17,22]), à l'exception de l'article de Brenier et al. [1], qui traite d'un système ayant une structure (cinétique) particulièrement riche ${ }^{2}$, ainsi que celui de Tzavaras [23], qui considère (2). Ce dernier traite aussi une relaxation plus simple, où $n=2$ mais le système relaxé a trois équations :

$$
\begin{aligned}
\partial_{t} u-\partial_{x} v & =0, \\
\partial_{t} v-\partial_{x} \sigma & =0, \\
\partial_{t}(\sigma-E u) & =-\frac{1}{\tau}(\sigma-g(u)) .
\end{aligned}
$$

La méthode de Tzavaras est basée sur la seule estimation d'énergie, de sorte que les solutions approchées peuvent ne pas rester bornées lorsque $\tau$ tend vers zéro. Il s'ensuit que le flux du système ( $g$ ou $f$ selon le cas) doit être globalement lipschitzien, sans quoi l'approximation n'est pas stable (la condition "sous-caractéristique" n'est plus vérifiée). Cette restriction limite bien entendu la portée du résultat.

Il existe principalement deux méthodes :

- l'une repose sur une propriété de contraction dans $L^{1}(\mathbb{R})$, qui est spécifique au cas scalaire car elle est liée à la théorie de Kružkhov,

- l'autre utilise la compacité par compensation, en suivant le canevas proposé par L. Tartar [21] et mis en œuvre par DiPerna [6].

On peut envisager d'étendre la seconde stratégie à des systèmes de plus grande taille, au moins ceux pour lesquels DiPerna a prouvé l'existence d'une solution du problème de Cauchy pour (1). C'est la voie suivie par Tzavaras, qui doit de plus utiliser les techniques développées par J. Shearer et Serre [20,19]; pour cette raison, il n'atteint qu'une partie des systèmes traités par DiPerna. C'est aussi l'idée de base du présent travail, ou l'on suit de plus près les techniques utilisées par DiPerna, avec des résultats de portée plus générales que dans [23]. Les théorèmes de convergence établis ici fournissent de nouvelles preuves de l'existence d'une solution entropique au problème de Cauchy pour (1) sous les mêmes hypothèses que dans [6]. Le problème de Cauchy pour des systèmes plus généraux reste cependant ouvert.

En résumé, on montre ici que, sous la condition "sous-caractéristique" (qui exprime la stabilité uniforme des états constants), l'existence de

\footnotetext{
${ }^{2}$ Grosso modo, le système à l'équilibre est constitué d'équations de Burgers $\partial_{t} u_{i}+$ $u_{i} \partial_{x} u_{i}=0$, qui ne sont couplées qu'à travers les chocs, par la condition de RankineHugoniot. Un cas particulier bien connu est celui d'un gaz isentropique de constante $\gamma=3$.
} 
domaines invariants convexes pour le système à l'équilibre entraîne la propriété analogue pour le système relaxé, bien que les uns soient de dimension $n$ et les autres de dimension $2 n$. En outre, toute entropie convexe de (1) s'étend en une entropie convexe "dissipative" de (2). Il ne semble pas que ces deux faits aient été notés auparavant, si ce n'est dans le cas scalaire. Par exemple, l'extension d'une entropie convexe n'avait été considérée qu'au voisinage de l'ensemble d'équilibre $\{(u, v) \in$ $\left.\mathcal{U} \times \mathbb{R}^{n} ; v=f(u)\right\}$ (voir [3]), ou bien lorsque (1) est scalaire (voir [9,14]). Au contraire, notre analyse est globale et valable pour $n \geqslant 1$.

Ces deux propriétés fournissent respectivement l'estimation a priori de $\left(u^{\tau}, v^{\tau}\right)$ dans $L^{\infty}$ et celle de $\tau^{-1 / 2}\left(f\left(u^{\tau}\right)-v^{\tau}\right)$ dans $L^{2}$. Utilisant alors l'estimation de la production d'entropie (comme dans [5]), ainsi que l'analyse de DiPerna [6] de la compacité par compensation (voir aussi les améliorations de l'auteur [18]), on en déduit un théorème de convergence, quand $\tau$ tend vers zéro, pour des données initiales bornées arbitraires. Ce résultat est un premier pas vers la validation des schémas numériques "par relaxation" de Jin et Xin, dont la convergence a été observée sur nombre de simulation numériques. Dans un travail en cours ([11], collaboration avec C. Lattanzio), on démontre la convergence de ces schémas sous des hypothèses analogues.

Pour des raisons historiques autant que pratiques, c'est d'abord la convergence de l'approximation (2) qui nous a intéressé. Cependant, comme l'a noté Natalini [16], elle n'est qu'un cas particulier d'une approximation plus générale, dans le style cinétique, où $\left(u^{\tau}, v^{\tau}\right)$ est remplacé par $z^{\tau}(x, t ; \xi)$, solution du système semi-linéaire

$$
\begin{aligned}
& \left(\partial_{t}+a(\xi) \partial_{x}\right) z^{\tau}=\frac{1}{\tau}\left(M_{\xi}\left(u^{\tau}\right)-z^{\tau}\right), \\
& u^{\tau}(x, t):=\int_{X} z^{\tau}(x, t ; \xi) d \mu(\xi) .
\end{aligned}
$$

Ici, l'espace de probabilité $(X, \Omega, \mu)$, les "Maxwelliennes" $\xi \mapsto M_{\xi}$ et les vitesses $a(\xi)$ sont fixés à l'avance, satisfaisant certaines conditions simples de consistance et de stabilité. Notre analyse s'applique aussi bien à ce type d'approximation. Le système (2) correspond au cas où $X$ n'a que deux éléments.

Le plan de cet article est le suivant. Connaissant des domaines invariants pour le système à l'équilibre, on en construit pour le système relaxé à la Section 2. L'énoncé clé est le Lemme 2.1, dont la démonstration, assez technique, est reportée à la fin de la section. On montre à la Section 3 que toute entropie convexe de (1) s'étend, de manière unique, en une 
entropie convexe de (2) qui, de plus, est dissipative. Les Théorèmes 2.1 et 3.1 sont les principaux résultats de ce travail, qui permettent de montrer la stabilité et la consistence de la relaxation semi-linéaire lorsque $\tau$ tend vers zéro. La Section 4 se contente de reprendre les techniques développées par les auteurs précédents pour en déduire la convergence, lorsque la stabilité et la consistance ont lieu. Quelques exemples de systèmes pour lesquels notre analyse prouve la convergence sont donnés. La dernière partie, enfin, indique comment cette stratégie s'adapte à l'approximation cinétique (3). On y dégage les hypothèses naturelles que doivent vérifier $(a, \mathcal{M}, \mu)$.

Je tiens à remercier C. Lattanzio, R. Natalini, M. Rascle et particulièrement A. Fathi pour les discussions fructueuses que j'ai eues avec eux sur ce sujet.

\section{DOMAINES POSITIVEMENT INVARIANTS}

Nous considérons des domaines convexes compacts $K \subset \mathcal{U}$, qui sont l'adhérence de leur intérieur. Le bord $\partial K$ est lipschitzien et on dit qu'il est caractéristique si l'espace tangent $T_{u} \partial K$ (défini en presque tout point) est stable par la différentielle $d f(u)$. La normale $v(u)$ à $\partial K$ est alors une forme linéaire propre de $d f(u)$, associée à une valeur propre $\lambda(u)$. Pour éviter des difficultés techniques inutiles, nous supposerons toujours que $\lambda(u)$ est une valeur propre simple. Le bord de $K$ est alors, par morceaux, une feuille intégrale du champ d'hyperplans $u+v(u)^{\perp}$. En particulier, il est de classe $\mathcal{C}^{2}$ par morceaux. A chacune de ces feuilles correspond un choix continu de la valeur propre $\lambda$.

La convexité de $K$ permet de définir, en tout point $s \in \partial K$, un cone convexe tangent $s+C(s)$, où $C(s)$ est l'ensemble des dérivées $u^{\prime}(0)$, lorsque $u$ parcourt l'ensemble des chemins différentiables $(u:[0,1] \rightarrow$ $K)$ tels que $u(0)=s$. Si $\partial K$ est caractéristique, le cone $C(s)$ est stable par $d f(s)$ : il suffit de le vérifier en tout point d'une partie dense de $\partial K$, par exemple aux points où $\partial K$ est de classe $\mathcal{C}^{2}$, ce qui est alors trivial.

L'existence de domaines convexes caractéristiques ne va pas de soi puisqu'elle requiert la condition de Frobenius $d l \wedge l \equiv 0$, où $l$ est une forme différentielle de degré 1 , propre pour $d f$. Cette condition est triviale pour $n \leqslant 2$, mais n'est que rarement satisfaite si $n \geqslant 3$. C'est l'une des raisons pour lesquelles l'étude du problème de Cauchy pour les systèmes d'au moins trois équations (en particulier celui qui décrit les ondes planes d'un gaz compressible) reste largement ouverte. 
Lemme 2.1. - Soit $K$ un convexe caractéristique, comme ci-dessus.

On suppose que

$$
a>\max \{\rho(d f(u)) ; u \in K\}
$$

(condition sous-caractéristique). Alors l'image $K_{+}$de $K$ par l'application $h_{+}: u \mapsto u+\frac{1}{a} f(u)$ est convexe, ainsi que l'image $K_{-}$de $K$ parl'application $h_{-}: u \mapsto u-\frac{1}{a} f(u)$. De plus, les applications $h_{ \pm}: K \rightarrow K_{ \pm}$ sont bijectives (ce sont donc des difféomorphismes).

Exemple. - Lorsque (1) est un système "de Temple" (voir [18]), ses domaines invariants compacts sont des polytopes, intersections de $2 n$ demi-espaces affines en général, donc affinement équivalents à des cubes. Dans ce cas, $K_{+}$et $K_{-}$sont encore des polytopes.

La démonstration du lemme, un peu longue, est reportée à la fin de cette section.

Lemme 2.2. - Sous les hypothèses du Lemme 2.1, on a

$$
K=\frac{1}{2}\left(K_{+}+K_{-}\right) .
$$

Ces deux lemmes ont les conséquences suivantes :

THÉORÈME 2.1. - Soit $K$ un convexe compact, positivement invariant pour (1). Supposons que

$$
a>\max \{\rho(d f(u)) ; u \in K\} .
$$

Soit $K_{ \pm}=h_{ \pm}(K)$ avec $h_{ \pm}(u):=u \pm \frac{1}{a} f(u)$. Alors l'ensemble

$$
D_{K}:=\left\{(u, v) ; u+\frac{1}{a} v \in K_{+} \text {et } u-\frac{1}{a} v \in K_{-}\right\}
$$

est positivement invariant pour le système relaxé (2).

COROllaire 2.1. - Soit $K$ un domaine comme au Théorème 2.1. Si $\left(u_{0}, v_{0}\right)$ est à valeurs dans $D_{K}$, alors le système relaxé (2) admet une et une seule solution $\left(u^{\tau}, v^{\tau}\right)$ définie et bornée sur $\mathbb{R}_{x} \times \mathbb{R}_{t}^{+}$, qui reste à valeurs dans $D_{K}$. De plus, $u^{\tau}$ est à valeurs dans $K$.

En particulier, si $u_{0}$ est à valeurs dans $K$ et si $v_{0}=f \circ u_{0}$ (donnée préparée), alors $u^{\tau}$ est à valeurs dans $K$. 
Preuve du Lemme 2.2. - Notons $C$ le convexe compact $\left(K_{+}+K_{-}\right) / 2$. Il sufit de montrer que pour toute forme linéaire non nulle $l$ sur $\mathbb{R}^{n}$, $\sup _{C} l=\sup _{K} l$. L'inégalité $\sup _{C} l \geqslant \sup _{K} l$ est une conséquence directe de $h_{-}(u)+h_{+}(u)=2 u$, qui implique $K \subset C$.

Par construction,

$$
\sup _{C} l=\frac{1}{2}\left(\sup _{K_{+}} l+\sup _{K_{-}} l\right) .
$$

Soit $\left(b_{+}+b_{-}\right) / 2$ un point de $C$ qui réalise $\sup _{C} l$, avec $b_{ \pm} \in K_{ \pm}$. Alors $b_{ \pm}$réalise $\sup _{K_{ \pm}} l$, c'est-à-dire que $l$ est une direction d'appui (sortante) de $K_{ \pm}$en $b_{ \pm}$. Notons $T_{ \pm}$les hyperplans d'appui correspondants : $T_{ \pm}=$ $b_{ \pm}+l^{\perp}$. Soit $b \in K$ tel que $h_{+}(b)=b_{+}$. La preuve du Lemme 2.1 montre que $T:=b+l^{\perp}$ est un hyperplan d'appui à $K$ en $b$ et aussi que (c'est le même argument) $h_{-}(b)+l^{\perp}$ est un hyperplan d'appui à $K_{-}$. Mais il n'y a qu'un seul hyperplan d'appui à $K_{-}$, dont la normale sortante soit $l$. On a donc $h(b)-b_{-} \in l^{\perp}$. Finalement $\sup _{C} l$ est atteint en $\left(b_{+}+h_{-}(b)\right) / 2$, c'est-à-dire en $b$. Ainsi $\sup _{C} l=l(b) \leqslant \sup _{K} l$.

Indiquons une autre preuve de $\left(K_{+}+K_{-}\right) / 2 \subset K$, lorsque $K$ est de plus invariant pour le problème de Riemann, ${ }^{3}$ dans lequel les chocs éventuels satisfont la condition de Lax affaiblie (avec des notations standard)

$$
\lambda_{1}\left(u_{d}\right) \leqslant \frac{d X}{d t} \leqslant \lambda_{n}\left(u_{g}\right) .
$$

Soit $\left(z_{+}+z_{-}\right) / 2$ un point de $\left(K_{+}+K_{-}\right) / 2$, avec $z_{ \pm}=h_{ \pm}\left(u_{ \pm}\right)$et $u_{ \pm} \in K$. Notons $U(x / t)$ la solution du problème de Riemann de $u_{+}$vers $u_{-}$, qui est à valeurs dans $K$.

Considérons le rectangle $[-\Delta x, \Delta x] \times] 0, \Delta t]$ du plan $\mathbb{R}_{x} \times \mathbb{R}_{t}$, avec $\Delta x=a \Delta t>0$. D'après (4) et la condition sous-caractéristique, $U$ vaut $u_{ \pm}$sur les cotés $x=\mp \Delta x$. Suivant un calcul classique, ${ }^{4}$ on intègre le système (1) sur le rectangle, pour obtenir

$$
\frac{1}{2}\left(z_{+}+z_{-}\right)=\frac{1}{2}\left(u_{+}+u_{-}\right)+\frac{\Delta t}{2 \Delta x}\left(f\left(u_{+}\right)-f\left(u_{-}\right)\right)
$$

\footnotetext{
${ }^{3}$ Les domaines invariants pour le problème de Riemann ont été étudiés par Hoff [8] ; grosso modo, ce sont les domaines convexes à bord caractéristiques.

${ }^{4}$ Utilisé notamment pour justifier le schéma de Lax-Friedrichs.
} 


$$
=\frac{1}{2 \Delta x} \int_{-\Delta x}^{\Delta x} U(x, \Delta t) d x
$$

Comme $U$ est à valeurs dans $K$, qui est convexe, cette moyenne appartient encore à $K$.

Preuve $d u$ Théorème 2.1. - Par définition, $D_{K}$ est caractéristique pour (2), puisque c'est un produit cartésien dans les coordonnées de Riemann $u \pm \frac{1}{a} v$. De plus, il est convexe. Comme enfin

$$
\begin{aligned}
& \left(\partial_{t}+a \partial_{x}\right)\left(u+\frac{1}{a} v\right)=\frac{1}{a \tau}(f(u)-v), \\
& \left(\partial_{t}-a \partial_{x}\right)\left(u-\frac{1}{a} v\right)=-\frac{1}{a \tau}(f(u)-v),
\end{aligned}
$$

il suffit de vérifier que le champ de vecteurs

$$
\left(u+\frac{1}{a} v, u-\frac{1}{a} v\right) \mapsto(f(u)-v, v-f(u))
$$

est rentrant dans $K_{+} \times K_{-}$. A savoir :

- pour $z_{0}=u+\frac{1}{a} v \in \partial K_{+}$et $z_{1}=u-\frac{1}{a} v \in K_{-}, f(u)-v$ est rentrant dans $K_{+}$,

- pour $z_{1} \in \partial K_{-}$et $z_{0} \in K_{+}, v-f(u)$ est rentrant dans $K_{-}$.

Voyons le premier cas (le second lui est symétrique). On a

$$
\frac{1}{a}(f(u)-v)=h_{+}(u)-z_{0},
$$

qui est bien rentrant car $u \in K$ (d'après le Lemme 2.2), $h_{+}(u) \in K_{+}$et $K_{+}$est convexe. De même pour le second cas :

$$
\frac{1}{a}(v-f(u))=h_{-}(u)-z_{1},
$$

qui est bien rentrant car $u \in K, h_{-}(u) \in K_{-}$et $K_{-}$est convexe.

Preuve du Corollaire 2.1. - Commençons par construire une solution approchée de (2) par la méthode de viscosité :

$$
\begin{aligned}
\partial_{t} u_{\varepsilon}+\partial_{x} v_{\varepsilon} & =\varepsilon \partial_{x}^{2} u_{\varepsilon}, \\
\partial_{t} v_{\varepsilon}+a^{2} \partial_{x} u_{\varepsilon} & =\varepsilon \partial_{x}^{2} v_{\varepsilon}+\frac{1}{\tau}(f(u)-v),
\end{aligned}
$$




$$
\left(u_{\varepsilon}, v_{\varepsilon}\right)(x, o)=\left(u_{0}, v_{0}\right)(x) .
$$

Ce problème de Cauchy admet une et une seule solution locale bornée. D'après la preuve du Théorème 2.1 , le domaine $D_{K}$ est positivement invariant pour ce système parabolique (voir [4]). La solution $\left(u_{\varepsilon}, v_{\varepsilon}\right)$ est donc globale en temps et à valeurs dans $D_{K}$. En particulier, $u_{\varepsilon}$ reste à valeurs dans $K$ (d'après le Lemme 2.2 ), de sorte que le système peut être vu comme un système à non-linéarité lipschitzienne.

On établit ensuite une estimation dans $C\left(0, T ; L_{\text {loc }}^{2}(\mathbb{R})\right)$, des expressions $R_{h} w_{\varepsilon}-w_{\varepsilon}$ et $R_{h} z_{\varepsilon}-z_{\varepsilon}$, où $R_{h}$ est une translation $x \mapsto x+h$ et $w, z=u \pm \frac{1}{a} v$. Cette estimation montre que $R_{h} w_{\varepsilon}-w_{\varepsilon}$ et $R_{h} z_{\varepsilon}-z_{\varepsilon}$ tendent vers zéro quand $h$ tend vers zéro, uniformément par rapport à $\varepsilon$. Ceci montre que $\left(u_{\varepsilon}, v_{\varepsilon}\right)_{\varepsilon>0}$ est une suite relativement compacte dans $L_{\text {loc }}^{p}\left(\mathbb{R} \times \mathbb{R}^{+}\right)$. Une valeur d'adhérence, quand $\varepsilon$ tend vers zéro, est alors une solution bornée de (2), à valeurs dans $D_{K}$ (utiliser à nouveau le fait que $f$ est lipschitzienne sur $K$ ).

Comme (2) est semi-linéaire, l'estimation d'énergie standard montre que cette solution bornée est unique.

\subsection{Démonstration du Lemme 2.1}

La condition sous-caractéristique assure que $h_{+}: K \rightarrow K_{+}$est un difféomorphisme local. En particulier, $\partial K_{+}$est inclus dans la variété $\Gamma_{+}:=h_{+}(\partial K)$ et celle-ci est immergée. Nous n'excluons pas a priori que $\Gamma_{+}$ait une auto-intersection, mais nous montrerons plus loin qu'il n'en est rien, c'est-à-dire que $\Gamma_{+}$est en fait plongée.

Montrons tout d'abord que, pour toute boule $B$ assez petite, centrée en un point $u_{0}$ de $\partial K$, l'image $\gamma_{+}$de $B \cap \partial K$ est le bord d'un convexe, celui-ci étant situé du même côté que l'image de $B \cap K$.

Commençons par le cas régulier : $\partial K$ est de classe $\mathcal{C}^{2}$ au voisinage de $u_{0}$. Soit $G(u) \leqslant 0$ une équation locale (i.e., dans $B$ ) de $K, G$ étant une submersion $\left(d G\left(u_{0}\right)\right.$ est non nul). Le bord étant caractéristique, $d G(u)$ est une forme propre de $d f$ le long de $\partial K: d G(u)(d f(u)-\lambda(u))=0$. Enfin, la convexité de $K$ s'exprime par

$$
D^{2} G_{u}(X, X) \geqslant 0, \quad u \in B \cap \partial K, \forall X \in d G(u)^{\perp} .
$$

Une équation locale de $h_{+}(B \cap K)$ est $g(z) \leqslant 0$, avec $G=g \circ h_{+}$. Comme $d G=\left(d g \circ h_{+}\right)\left(I+\frac{1}{a} d f\right)$ et $d G$ est propre, on tire $d G=\mu d g \circ h_{+}$, avec $\mu=1+\lambda / a>0$. Ainsi, l'espace tangent en $h_{+}\left(u_{0}\right)$ à $\gamma_{+}$est parallèle à l'espace tangent en $u_{0}$ à $\partial K$. Différenciant une seconde fois le long de $\partial K$, il vient, pour tout $Y \in T_{u} \partial K$ et $X \in \mathbb{R}^{n}$ : 


$$
\begin{aligned}
& D^{2} G(X, Y) \\
& \quad=(d \mu \cdot Y) d g \circ h_{+} \cdot X+\mu D^{2} g \circ h_{+}\left(X,\left(I+\frac{1}{a} d f\right) Y\right) .
\end{aligned}
$$

Pour $X, Y \in T_{u} \partial K=d G(u)^{\perp}$, cela se réduit à

$$
D^{2} G(X, Y)=\mu D^{2} g \circ h_{+}\left(X,\left(I+\frac{1}{a} d f\right) Y\right) .
$$

En termes matriciels (on choisit une base de $T_{u} \partial K$ ), cela s'écrit $\Sigma=$ $S A$, où $\Sigma$ et $S$ sont symétriques, $\Sigma$ est semi-définie positive et $A$ est diagonalisable à valeurs propres réelles strictement positives. On en déduit classiquement que $S \geqslant 0$, c'est-à-dire que la restriction de $D^{2} g$ à $T_{h_{+}(u)} \gamma_{+}$est semi-définie positive : $\gamma_{+}$est donc bien le bord d'un convexe, situé du même côté que $h_{+}(B \cap K)$.

De même, lorsque $u_{0}$ est un point singulier de $\partial K, h_{+}\left(u_{0}\right)$ est aussi un point singulier de $\gamma_{+}$. Comme le cône convexe $C\left(u_{0}\right)$ est invariant par $d f\left(u_{0}\right), \gamma_{+}$admet un cône tangent en $h_{+}\left(u_{0}\right)$, égal à $h_{+}\left(u_{0}\right)+C\left(u_{0}\right)$, qui est donc convexe.

Finalement, le tenseur de courbure le long de $\gamma_{+}$, qui peut être à valeurs mesures (à cause des singularités), est positif partout et $\gamma_{+}$est bien le bord d'un convexe, celui-ci étant situé du même côté que $h_{+}(B \cap K)$.

Montrons maintenant que $\Gamma_{+}$est bien le bord de $\partial K_{+}$. Celui-ci est un compact. Etant donné une forme linéaire $l \neq 0$, il existe donc un hyperplan d'appui $\Pi_{l}$ à $K_{+}$, dont la normale sortante est $l$. Soit $z_{0}$ un point d'appui de $\Pi_{l}$. Comme $\partial K_{+} \subset \Gamma_{+}$, on a $z_{0}=h_{+}\left(u_{0}\right)$ pour un $u_{0} \in \partial K$. Ce qui précède montre que $K$ admet en $u_{0}$ un hyperplan d'appui $\pi_{l}$ parallèle à $\Pi_{l}$. Comme $K$ est convexe, cette propriété caractérise $\pi_{l}$ de manière unique. En revanche, $u_{0}$ peut ne pas être unique, si $\pi_{l}$ s'appuie sur $K$ le long d'un convexe $K_{0}$; mais dans ce cas, l'image $h_{+}\left(K_{0}\right)$ est incluse dans $\Pi_{l}$. En effet, si $\left[u_{0}, u_{1}\right]$ est inclus dans $K_{0}$, alors $l\left(u_{1}-u_{0}\right)=0$ et $h_{+}\left(\left[u_{0}, u_{1}\right]\right)$ est une courbe qui est partout tangente à $l$. Finalement, $\Pi_{l}$ est unique lui aussi.

Cette analyse montre que l'ensemble des hyperplans d'appui de $\partial K_{+}$ comprend tous les espaces tangents à $\Gamma_{+}$, ce qui implique $\Gamma_{+} \subset \partial K_{+}$, c'est-à-dire $\Gamma_{+}=\partial K_{+}$. En particulier, $\Gamma_{+}$n'a pas d'auto-intersection : tous ces points sont exposés. C'est donc le bord d'un convexe, situé du même côté de $\Gamma_{+}$que $K_{+}$. Mais comme $\partial K_{+}=\Gamma_{+}$, ce convexe est en fait $K_{+}$. Enfin, $h_{+}$, restreinte à $\partial K$, est injective (parce que localement injective et qu'il n'y a pas d'auto-intersection). 
Il reste à montrer que $h_{+}: K \rightarrow K_{+}$est bijectif. Pour cela, nous considérons l'homotopie $h_{\varepsilon}(u):=u+\varepsilon f(u)$, pour $0 \leqslant \varepsilon \leqslant 1 / a$. Notant $K_{\varepsilon}=h_{\varepsilon}(K)$, ce qui précède s'applique pour chaque $\varepsilon$ :

(1) $K_{\varepsilon}:=h_{\varepsilon}(K)$ est convexe et son bord est $h_{\varepsilon}(\partial K)$,

(2) l'intérieur de $K_{\varepsilon}$ est égal à l'image de l'intérieur de $K$,

(3) $h_{\varepsilon}: K \rightarrow K_{\varepsilon}$ est un difféomorphisme local,

(4) $h_{\varepsilon}$, restreinte à $\partial K$, est injectif.

Bien entendu, $h_{1 / a}$ et $K_{1 / a}$ coïncident avec $h_{+}$et $K_{+}$.

Tout d'abord, si $z \in \partial K_{+}$, l'image réciproque de $z$ par $h_{+}$est contenue dans $\partial K$, car $h_{+}$est une application ouverte. D'après 1 et 4 , l'équation $h_{+}(u)=z$ admet donc une et une seule solution.

Il reste le cas où $z$ est intérieur à $K_{+}$. D'après $2, z=h_{+}\left(u_{0}\right)$ pour au moins un $u_{0}$ intérieur à $K$. Définissons alors la courbe (un segment, en fait) $\varepsilon \mapsto z(\varepsilon):=h_{\varepsilon}\left(u_{0}\right)$. Pour tout $\varepsilon \in[0,1 / a], z(\varepsilon)$ est intérieur à $K_{\varepsilon}$. Comme $h_{\varepsilon}$ n'a que des valeurs régulières (d'après 3 ), $z(\varepsilon)$ en est une. Comme, de plus, l'équation $h_{\varepsilon}(u)=z(\varepsilon)$ n'a pas de solution sur $\partial K$ (à cause de 1 ), le cardinal $m(\varepsilon)$ de $h_{\varepsilon}^{-1}(z(\varepsilon))$ est constant. Ainsi, $m(1 / a)=m(0)$, qui vaut 1 car $h_{0}=\mathrm{id}_{K}$. Ainsi $h_{+}$est bijectif.

Les énoncés concernant $h_{-}$et $K_{-}$s'obtiennent simplement en remplaçant $f$ par $-f$. Ceci achève la preuve du Lemme 2.1.

\section{EXTENSION DES ENTROPIES CONVEXES}

Nous nous plaçons dans le cadre de la section précédente : $K$ est un domaine invariant pour (1) et $D_{K}$ est le domaine correspondant pour (2). On se donne maintenant une paire entropie-flux $(\eta, q)$ pour le système à l'équilibre et on cherche une paire $(E, F)$, entropie-flux pour le système relaxé, qui coïncide avec $(\eta, q)$ sur l'ensemble d'équilibre, c'est-à-dire qui satisfait

$$
(E, F)(u, f(u))=(\eta, q)(u), \quad u \in K .
$$

Naturellement, nous nous sommes restreints au domaine $K$, sur lequel on peut s'assurer de la condition sous-caractéristique. En particulier, $h_{ \pm}: K \rightarrow K_{ \pm}$sont des homéomorphismes.

L'entropie générale de (2) est de la forme

$$
\begin{aligned}
& E(u, v)=e_{+}\left(u+\frac{1}{a} v\right)+e_{-}\left(u-\frac{1}{a} v\right), \\
& F(u, v)=a e_{+}\left(u+\frac{1}{a} v\right)-a e_{-}\left(u-\frac{1}{a} v\right) .
\end{aligned}
$$


La coïncidence (6) est donc réalisée si et seulement si

$$
2 e_{+} \circ h_{+}(u)=\eta(u)+\frac{1}{a} q(u), \quad 2 e_{-} \circ h_{-}(u)=\eta(u)-\frac{1}{a} q(u),
$$

ce qui définit $e_{ \pm}$de manière unique sur $K_{ \pm}$. Ainsi, le couple $(E, F)$ est défini de manière unique sur $D_{K}$ :

Proposition 3.1. - Etant donnée une paire entropie-flux $(\eta, q)$ : $K \rightarrow \mathbb{R}^{2}$ de (1), il existe une et une seule paire entropie-flux $(E, F)$ : $D_{K} \rightarrow \mathbb{R}^{2}$ de (2) qui cö̈ncide avec $(\eta, q)$ sur l'ensemble d'équilibre (le graphe de $f$ ). Cette paire est définie par les formules (7).

De plus, si $\eta$ est convexe (resp. strictement convexe), alors $E$ est convexe (resp. strictement).

Il reste à montrer la convexité. Pour cela, différencions (7) pour obtenir

$$
\left(d e_{ \pm}\right) \circ h_{ \pm}=\frac{1}{2} d \eta
$$

Différencions encore une fois :

$$
D^{2} e_{ \pm}\left(X \pm \frac{1}{a} d f(u) X, Y\right)=\frac{1}{2} D^{2} \eta(X, Y), \quad X, Y \in \mathbb{R}^{n} .
$$

En termes matriciels, cela s'écrit $\Sigma A=S$, où $\Sigma$ est symétrique, $S$ est symétrique (définie) positive et $A$ est diagonalisable à valeurs propres réelles strictement positives. Il s'ensuit que $\Sigma$ est (définie) positive, ce qui exprime la convexité (stricte) de $e_{ \pm}$, donc celle de $E$.

THÉORÈME 3.1. - Si l'entropie $\eta$ est convexe sur K, alors l'entropie E convexe qui lui est associée est dissipative sur $D_{K}$, c'est-à-dire

$$
d_{v} E(u, v) \cdot(f(u)-v) \leqslant 0, \quad \forall(u, v) \in D_{K} .
$$

En fait, si $D^{2} \eta>0$ sur $K$, il existe un nombre $\alpha>0$ tel que

$$
d_{v} E(u, v) \cdot(f(u)-v) \leqslant-\alpha\|f(u)-v\|^{2}, \quad \forall(u, v) \in D_{K} .
$$

Démonstration. - Comme $E$ est convexe sur $D_{K}$, sa différentielle est monotone et nous avons

$$
\begin{aligned}
& d_{v} E(u, v) \cdot(f(u)-v)+\alpha\|f(u)-v\|^{2} \\
& \quad \leqslant d_{v} E(u, f(u)) \cdot(f(u)-v),
\end{aligned}
$$


où $\alpha \geqslant 0$ est tel que $D^{2} E \geqslant \alpha I_{2 n}$ sur $D_{K}$. On a $\alpha>0$ si $D^{2} \eta>0$ sur $K$, d'après la Proposition 3.1. Comme $d_{v} E$ s'annule le long du graphe de $f$, le second membre est nul et on a l'inégalité annoncée.

A nouveau, voici une démonstration amusante lorsque $K$ est invariant pour le problème de Riemann. Comme $E$ est convexe, nous avons

$$
d_{v} E(u, v) .(f(u)-v)+\frac{\alpha}{2}\|f(u)-v\|^{2} \leqslant E(u, f(u))-E(u, v) .
$$

Il reste donc à montrer que $\eta(u)-E(u, v)$ est négatif.

Pour cela, notons $z_{ \pm}:=u \pm \frac{1}{a} v \in K_{ \pm}$. On a $z_{+}=h_{+}\left(u_{g}\right)$ et $z_{-}=$ $h_{-}\left(u_{d}\right)$, avec $u_{g, d} \in K$. Soit $U(x / t)$ la solution du problème de Riemann entre $u_{g}$ et $u_{d}$, à valeurs dans $K$. Intégrant l'inégalité $\partial_{t} \eta(U)+\partial_{x} q(U) \leqslant$ 0 sur le rectangle $[-\Delta x, \Delta x] \times] 0, \Delta t]$ comme précédemment, ${ }^{5}$ nous obtenons

$$
\int_{-\Delta x}^{\Delta x} \eta \circ U(x, \Delta t) d x \leqslant \Delta x\left(\eta\left(u_{g}\right)+\eta\left(u_{d}\right)\right)+\Delta t\left(q\left(u_{d}\right)-q\left(u_{g}\right)\right) .
$$

Comme

$$
u=\frac{1}{2 \Delta x} \int_{-\Delta x}^{\Delta x} U(x, \Delta t) d x
$$

on déduit avec l'inégalité de Jensen ${ }^{6}$ que

$$
\eta(u) \leqslant \frac{1}{2}\left(\eta\left(u_{g}\right)+\eta\left(u_{d}\right)\right)+\frac{1}{2 a}\left(q\left(u_{d}\right)-q\left(u_{g}\right)\right),
$$

c'est-à-dire $\eta(u) \leqslant E(u, v)$.

COROllaire 3.1. - On suppose que le système (1) admet un domaine convexe compact invariant $K$ et une entropie $\eta$ vérifiant $D^{2} \eta>0$ sur K. On suppose aussi que

$$
a>\max \{\rho(d f(u) ; u \in K\} .
$$

\footnotetext{
${ }^{5}$ Ici, nous supposons que les chocs éventuels dans la solution du problème de Riemann satisfont l'inégalité d'entropie de Lax pour l'entropie convexe $\eta$.

${ }^{6}$ Ici, nous pouvons récupérer un terme positif $\beta\|f(u)-v\|^{2}$, car l'inégalité de Jensen est vraie avec $\alpha_{\eta}\left\|u_{d}-u_{g}\right\|^{2}$ au premier membre. Or $u_{d}$ n'est égal à $u_{g}$ que si $v=f(u)$.
} 
Soit $\left(u_{0}, v_{0}\right)$ une donnée initiale pour (2), à valeurs dans $D_{K}$. Alors la solution $\left(u^{\tau}, v^{\tau}\right)$ de (2) satisfait l'estimation

$$
\frac{\alpha}{\tau} \iint_{(-L, L) \times(0, T)}\|f(u)-v\|^{2} d x d t \leqslant C(L, T),
$$

où $C(L, T)$ est indépendant de $\tau>0$.

Démonstration. - Soit $E$ l'entropie associée à $\eta$ et soit $\phi \in \mathcal{D}(\mathbb{R})$ une fonction test positive, telle que $\phi(x) \equiv 1$ pour $|x|<L$. Alors

$$
\frac{d}{d t} \int_{\mathbb{R}} \phi(x) E\left(u^{\tau}, v^{\tau}\right) d x+\frac{\alpha}{\tau} \int_{\mathbb{R}}\left\|f\left(u^{\tau}\right)-v^{\tau}\right\|^{2} d x=\int_{\mathbb{R}} \phi^{\prime} F d x .
$$

Le second membre est majoré par une constante $c_{\phi, K}$, grâce à l'estimation $L^{\infty}$ de $\left(u^{\tau}, v^{\tau}\right)$. Il reste à intégrer sur $(0, T)$ pour obtenir l'inégalité annoncée.

\section{CONVERGENCE}

Cette section reprend sans changement les techniques développées par les auteurs précédents $[2,3,14,5]$.

Etant donné un domaine invariant $K$ pour (1), la condition souscaractéristique et le domaine $D_{K}$, qui est invariant pour (2), on considère une donnée initiale $\left(u_{0}, v_{0}\right)$ à valeurs dans $D_{K}$. En particulier, $u_{0}$ est à valeurs dans $K$ et, réciproquement, $\left(u_{0}, f \circ u_{0}\right)$ est à valeurs dans $D_{K}$ si et seulement si $u_{0}$ est à valeurs dans $K$.

La solution unique $\left(u^{\tau}, v^{\tau}\right)$ du problème de Cauchy pour (2) est à valeurs dans $D_{K}$. On en déduit que $u^{\tau}$ est à valeurs dans $K$, donc reste dans un borné de $L^{\infty}\left(\mathbb{R} \times \mathbb{R}^{+}\right)$quand $\tau$ tend vers zéro. De plus, $\tau^{-1 / 2}\left(f\left(u^{\tau}\right)-v^{\tau}\right)$ reste dans un borné de $L^{2}\left(\mathbb{R} \times \mathbb{R}^{+}\right)$. Quitte à extraire une sous-suite, nous supposons désormais que $u^{\tau}$ converge dans $L^{\infty}$ faible-étoile, ainsi que toute les suites $g \circ u^{\tau}$ pour $g \in \mathcal{C}^{0}(K)$. Nous noterons $v_{x, t}$ la mesure de Young associée, définie par

$$
\left(\lim _{\tau \rightarrow 0+} g \circ u^{\tau}\right)(x, t)=\left\langle v_{x, t}, g\right\rangle, \quad \text { p.p. }(x, t) \in \mathbb{R} \times \mathbb{R}^{+} .
$$

Considérons maintenant une paire entropie-flux $(\eta, q)$ de (1), qu'on étend en une paire entropie-flux $(E, F)$ de (2). Le long de la courbe 
d'équilibre, on a

$$
d_{v} E(u, f(u))=\frac{1}{a}\left(d e_{+} \circ h_{+}-d e_{-} \circ h_{-}\right)=\frac{1}{a}(d \eta-d \eta)=0,
$$

de sorte que $\left\|d_{v} E\right\| \leqslant c_{0}\|f(u)-v\|$ dans $D_{K}$. La dissipation d'entropie pour $u^{\tau}$ se décompose alors sous la forme

$$
\begin{aligned}
& \partial_{t} \eta\left(u^{\tau}\right)+\partial_{x} q\left(u^{\tau}\right) \\
& \quad=\partial_{t}(\eta-E)+\partial_{x}(q-F)+\frac{1}{\tau} d_{v} E \cdot(f(u)-v) .
\end{aligned}
$$

Le premier membre de (9) est dans un borné de $W^{-1, \infty}$, puisque $u^{\tau}$ est borné dans $L^{\infty}$. Le second membre consiste en deux types de termes. D'une part $\partial_{t}(\eta-E)+\partial_{x}(q-F)$; ces termes tendent vers zéro dans $H^{-1}$, comme $\tau^{1 / 2}$, car il en est de même pour $\eta-E$ et $q-F$ dans $L^{2}$. En effet, $\eta-E=E\left(u^{\tau}, f\left(u^{\tau}\right)\right)-E\left(u^{\tau}, v^{\tau}\right)$ et $E(u, f(u))-$ $E(u, v)=\mathcal{O}(f(u)-v)$ uniformément sur le compact $D_{K}$. D'autre part, $\frac{1}{\tau} d_{v} E \cdot(f(u)-v)$ est majoré par $c_{0} \tau^{-1}\|f(u)-v\|^{2}$, qui est borné dans $L^{1}$. Le lemme de Murat [13] assure alors que la suite $\left(\partial_{t} \eta\left(u^{\tau}\right)+\right.$ $\left.\partial_{x} q\left(u^{\tau}\right)\right)_{\tau>0}$ reste dans un compact de $H_{l o c}^{-1}$. On peut donc appliquer le lemme divergence-rotatiomel de la compacité par compensation, comme dans [21].

On obtient ainsi l'identité de Tartar, pour presque tout $(x, t)$ et pour tout couple de paires entropie-flux $\left(\eta_{j}, q_{j}\right)_{j=1,2}$ :

$$
\left\langle v, \eta_{1} q_{2}-\eta_{2} q_{1}\right\rangle=\left\langle v, \eta_{1}\right\rangle\left\langle v, q_{2}\right\rangle-\left\langle v, \eta_{2}\right\rangle\left\langle v, q_{1}\right\rangle
$$

On peut alors conclure comme DiPerna [6] à la convergence forte de $u^{\tau}$ dans $L_{l o c}^{p}$, pourvu que le système (1) ait assez d'entropies (i.e., qu'il soit riche) et que les champs caractéristiques soient vraiment non-linéaires, avec éventuellement un point d'inflexion. Le théorème final est le suivant.

THÉORÈME 4.1. - On suppose que le système (1) a les propriétés requises par DiPerna pour la convergence de la méthode de viscosité artificielle:

- il existe un domaine compact convexe $K$ à bord caractéristique et de classe $\mathcal{C}^{2}$ par morceaux,

- il existe une entropie $\eta_{0}$ telle que $D^{2} \eta_{0}>0$ sur $K$,

- les seules probabilités $v$, à support dans $K$, solutions de l'équation de Tartar, sont les masses de Dirac. 
On impose enfin la condition sous-caractéristique :

$$
a>\max \{\rho(d f(u)) ; u \in K\} .
$$

Alors, pour tout $\left(u_{0}, v_{0}\right) \in L^{\infty}(\mathbb{R})^{2}$, à valeurs dans $D_{K}$ (en particulier si $u_{0}$ est à valeurs dans $K$ et $v_{0}=f \circ u_{0}$ ), le système relaxé (2) admet une unique solution bornée, définie sur $\mathbb{R} \times \mathbb{R}^{+}$. De plus,

(1) $u^{\tau}$ converge dans $L_{\text {loc }}^{1}$ fort vers une solution entropique $u$ de (1), à valeurs dans $K$,

(2) $v^{\tau}$ converge également, vers $f(u)$.

\subsection{Applications}

Les systèmes les plus simples auquels le Théorème 4.1 s'applique sont ceux dits "de Temple". Ces systèmes sont munis d'invariants de Riemann forts (satisfaisant $d w_{j}\left(d f-\lambda_{j}\right)=0$ ), dont les ensembles de niveau sont des hyperplans. Les domaines

$$
K_{\alpha, \beta}:=\left\{u \in \mathcal{U} ; \alpha_{j} \leqslant w_{j}(u) \leqslant \beta_{j}\right\}
$$

sont des compacts convexes (comme intersections de demi-espaces) invariants (puisque caractéristiques). L'analyse de l'équation de Tartar (10) est très complète, grâce à l'existence d'entropies "à la Kružkhov" (voir [7]).

En second lieu, on peut appliquer le Théorème 4.1 au système de l'élasticité

$$
\partial_{t} u_{1}+\partial_{x} u_{2}=0, \quad \partial_{t} u_{2}+\partial_{x} p\left(u_{1}\right)=0,
$$

lorsque $p^{\prime}>0$ et $s p^{\prime \prime}(s)>0$ pour $s \neq 0$. DiPerna a montré que l'équation de Tartar n'a pas d'autres solutions que les masses de Dirac. Il utilise en outre une famille absorbante de domaines invariants compacts, de la forme

$$
K_{b, R}:=\left\{u \in \mathbb{R}^{2} ;\left|g\left(u_{1}\right)\right|+\left|u_{2}-b\right| \leqslant R\right\},
$$

où $g^{\prime}=\sqrt{p^{\prime}}$. L'energie mécanique

$$
\eta_{0}:=\frac{1}{2} u_{2}^{2}+\int_{0}^{u_{1}} p(s) d s
$$

convient pour l'estimation de la dissipation. 


\section{LES MODÈLES CINÉTIQUES DE NATALINI*}

Dans son article [16], Natalini propose d'approcher la solution d'une équation scalaire de la forme (1) par celle du système semi-linéaire

$$
\partial_{t} z_{i}^{\tau}+c_{i} \partial_{x} z_{i}^{\tau}=\frac{1}{\tau}\left(M_{i}\left(u^{\tau}\right)-z_{i}^{\tau}\right), \quad 1 \leqslant i \leqslant N,
$$

où

$$
u^{\tau}:=\sum_{i=1}^{N} z_{i}^{\tau} .
$$

Les fonctions $M_{i}$ sont des "Maxwelliennes", données a priori, de même que les vitesses $c_{i}$. Elles satisfont des conditions de consistance et de stabilité que nous ne détaillerons pas puisque ce sont des cas particuliers de ce qui suit. Le lien avec l'approximation (2) est le suivant : si $N=2$ et $c_{i}=(-1)^{i} a$, la consistance implique le choix $M_{1}=h_{-} / 2, M_{2}=h_{+} / 2$. Alors (11) équivaut à (2), via le changement de variable $\left(z_{1}, z_{2}\right) \mapsto(u=$ $\left.z_{1}+z_{2}, v=a\left(z_{2}-z_{1}\right)\right)$.

Bien entendu, le modèle de Natalini a un intérêt pour l'approximation des systèmes et pas seulement pour celle des équations scalaires. En outre, il n'y a aucune raison de se limiter à un modèle discrêt, dans lequel $z(x, t)$ est à valeurs dans un espace de dimension finie $n N$. Il y a même un intérêt à recourir à un espace de vitesses infini : c'est dans ce câdre que des théorèmes de compacité en moyenne sont disponibles. Nous considérons donc le modèle plus général

$$
\begin{aligned}
& \left(\partial_{t}+a(\xi) \partial_{x}\right) z^{\tau}=\frac{1}{\tau}\left(M_{\xi}\left(u^{\tau}\right)-z^{\tau}\right), \\
& u^{\tau}(x, t):=\int_{X} z^{\tau}(x, t, \xi) d \mu(\xi) .
\end{aligned}
$$

Ici, $(X, \Omega, \mu)$ est un espace de probabilité et $a: X \rightarrow \mathbb{R}$ est mesurable bornée. On ne perd rien à supposer que $X$ est le support de $\mu$. Les Maxwelliennes $M_{\xi}$ sont des fonctions de classe $\mathcal{C}^{2}$ sur un domaine convexe compact $K$, mesurables par rapport à $\xi$. Les conditions de consistance sont

\footnotetext{
* Depuis la soumission de cet article, F. Bouchut a développé le même formalisme que dans cette section, mais pour des motifs différents des nôtres. Voir "Construction of BGK models with a family of kinetic entropies for a given system of conservation laws". J. Statist. Phys. 95 (1999) 113-170.
} 
H1 Pour tout $s$ dans $K: \int_{X} M_{\xi}(s) d \mu(\xi)=s$,

H2 Pour tout $s$ dans $K: \int_{X} a(\xi) M_{\xi}(s) d \mu(\xi)=f(s)$.

On espère que l'équilibre $z(x, t, \xi) \sim M_{\xi}(u(x, t))$ est atteint lorsque $\tau$ tend vers zéro. Comme on a

$$
\partial_{t} u^{\tau}+\partial_{x} \int_{X} a(\xi) z^{\tau}(\xi) d \mu(\xi)=0,
$$

on aura $\partial_{t} u+\partial_{x} f(u)=0$ si la convergence a lieu dans un sens assez fort.

Les hypothèses qui permettent d'obtenir la stabilité $L^{\infty}$ généralisent ${ }^{7}$ celles de Natalini :

H3 Le domaine $K$ est invariant pour le système (1).

H4 Pour tout $(s, \xi)$ dans $\left.K \times X: \operatorname{Sp}\left(d M_{\xi}\right) \subset\right] 0,+\infty[$.

H5 Pour tout $(s, \xi)$ dans $K \times X$, le commutateur $\left[d M_{\xi}(s), d f(s)\right]$ est nul.

Notons $\lambda_{j}(s)(1 \leqslant j \leqslant n)$ les valeurs propres de $d f(s)$, qui sont simples par hypothèse. Notons $r_{j}(s)$ des vecteurs propres associés et $l_{j}(s)$ des formes linéaires propres : $l_{j} \cdot r_{k} \equiv \delta_{j}^{k}$. La condition (H5) permet d'écrire $d M_{\xi}(s) r_{j}(s)=\rho_{j}(\xi, s) r_{j}(s)$. Les hypothèses $(\mathbf{H 1}, \mathbf{H 2}, \mathbf{H 4})$ impliquent respectivement

(1) $\int_{X} \rho_{j}(\xi, s) d \mu(\xi)=1$,

(2) $\int_{X} a(\xi) \rho_{j}(\xi, s) d \mu(\xi)=\lambda_{j}(s)$,

(3) $\rho_{j}(\xi, s)>0$.

En particulier, on en déduit la propriété "sous-caractéristique"

$$
\forall s \in K, \quad \operatorname{Sp} d f(s) \subset\left[\inf _{X} a, \sup _{X} a\right] .
$$

L'hypothèse (H5) est très contraignante et on peut craindre quelle limite le choix des Maxwelliennes à des combinaisons linéaires du flux $f$ et de l'identité, donnant ainsi une assez pauvre généralisation de la relaxation. Cette crainte est justifiée pour des systèmes généraux d'au moins trois équations, en particulier pour le système complet de la dynamique des gaz. Cependant, l'hypothèse $(\mathbf{H 3})$ a déjà limité le choix des exemples : essentiellement, ce sont les systèmes dits "riches", dits aussi semi-hamiltoniens (voir [18]). Cette classe contient tous les systèmes $2 \times 2$, ainsi que nombre d'autres issus de la chromatographie, de l'électrophorèse, ... Pour cette classe, le système d'EDPs sur-déterminé

\footnotetext{
${ }^{7}$ En fait, (H3, H5) sont triviales dans le cas scalaire.
} 
(H5) admet un très grand nombre de solutions, qui forment un espace vectoriel isomorphe à $\mathcal{C}^{2}(\mathbb{R})^{n}$. Il y a des solutions, combinaisons linéaires de $f$ et de l'identité, qui satisfont de plus (H4); enfin les solutions qui leurs sont proches satisfont encore (H4). Il y a donc une grande liberté dans le choix de l'approximation cinétique de ces systèmes.

Remarque. - Notons, ce qui sera important plus loin, que les fonctions $M: K \rightarrow \mathbb{R}^{n}$ qui satisfont (H5) sont les flux pour lesquels le flot du système $\partial_{t} v+\partial_{x} M(v)=0$ commute avec le flot de (1), tant que les solutions restent de classe $\mathcal{C}^{1}$. Ce sont aussi des flux pour lesquels ces systèmes ont les mêmes entropies que (1) (mais pas les mêmes flux d'entropie).

Solutions locales. - Le problème de Cauchy pour (12) est résolu par une méthode de point fixe, en utilisant le semi-groupe de l'équation linéaire $\partial_{t} z+a(\xi) \partial_{x} z=0$ et la formule de Duhamel. On obtient ainsi une solution locale en temps, aussi régulière en $(x, t)$ que la donnée initiale. Pour que cette solution soit globale, il suffit qu'elle reste uniformément bornée, ce qui sera le cas si on dispose d'un domaine positivement invariant pour (12). C'est bien le cas, grâce aux hypothèses ci-dessus. En effet, le Lemme 2.1 reste valable lorsqu'on remplace $h_{ \pm}$par $M_{\xi}$, de sorte que les domaines $K_{\xi}:=M_{\xi}(K)$ sont convexes. Puis le Lemme 2.2 admet la généralisation suivante, avec une démonstration semblable :

Proposition 5.1. - Si $\xi \mapsto w(\xi)$ est mesurable sur $X$, avec $w(\xi) \in$ $K_{\xi}$, alors

$$
\int_{X} w(\xi) d \mu(\xi) \in K .
$$

Le domaine utile $D$ pour (12) est donc l'ensemble des applications mesurables $\xi \mapsto w(\xi)$ telles que $w(\xi) \in K_{\xi}$ presque partout. Lorsque $X$ est fini, $D$ est isomorphe à $\prod_{\xi \in X} K_{\xi}$. C'est un convexe positivement invariant pour (12) car, lorsque $z \in D$ et $z\left(\xi_{0}\right) \in \partial K_{\xi_{0}}$, le vecteur

$$
\frac{1}{\tau}\left(M_{\xi_{0}}(u)-z\left(\xi_{0}\right)\right)
$$

est rentrant. En effet, $u \in K$ d'après la Proposition 5.1, de sorte que $M_{\xi_{0}}(u) \in K_{\xi_{0}}$. On peut démontrer l'invariance de $D$ pour (12) en suivant la stratégie de [4]. On obtient donc le résultat suivant : 
THÉORÈme 5.1. - Sous les hypothèses $(\mathbf{H 1}, \mathbf{H 2}, \mathbf{H 3}, \mathbf{H 4}, \mathbf{H 5})$ et si la condition initiale $z_{0}: \mathbb{R} \rightarrow D$ est mesurable, le problème de Cauchy pour (12) admet une et une seule solution globale $z: \mathbb{R} \times \mathbb{R}^{+} \rightarrow D$.

Après la stabilité $L^{\infty}$, nous nous tournons vers la convergence de $M_{\xi}\left(u^{\tau}\right)-z^{\tau}(\xi)$. Pour cela, nous construisons des paires entropie-flux $(E, F)$ pour (12). Si $(\eta, q)$ est une paire entropie-flux de (1), on désire que $(E[w], F[w])$ vaille $(\eta(u), q(u))$ lorsque $w(\xi)=M_{\xi}(u)$ pour tout $\xi$. Comme $(E, F)$ est de la forme

$$
E[w]:=\int_{X} e_{\xi}(w(\xi)) d \mu(\xi), \quad F[w]:=\int_{X} a(\xi) e_{\xi}(w(\xi)) d \mu(\xi),
$$

il suffit de poser, grâce à (H1, H2) :

$$
e_{\xi}=q_{\xi} \circ M_{\xi}^{-1},
$$

où $q_{\xi}$ est le flux de l'entropie $\eta$ pour le système $\partial_{t} v+\partial_{x} M_{\xi}(v)=0$ (voir la remarque ci-dessus). Ce flux est défini à une constante additive près. On a alors, si $w(\xi)=M_{\xi}(u)$ :

$$
E[w]=\int_{X} e_{\xi} \circ M_{\xi}(u) d \mu(\xi)=\int_{X} q_{\xi}(u) d \mu(\xi) .
$$

Cette dernière intégrale est le flux d'entropie de $\eta$ pour le système $\partial_{t} v+\partial_{x} h(v)=0$, avec

$$
h:=\int_{X} M_{\xi} d \mu(\xi)
$$

D'après $(\mathbf{H 1}), h$ est l'identité, donc $E[w]=\eta(u)$, à une constante près qu'on peut prendre nulle par un choix convenable des $q_{\xi}$. De même, on obtient $F[w]=q(u)$.

En différenciant, il vient

$$
d e_{\xi} \circ M_{\xi}=d \eta, \quad\left(D^{2} e_{\xi} \circ M_{\xi}\right) d M_{\xi}=D^{2} \eta .
$$

Lorsque $\eta$ est convexe, nous obtenons à nouveau que $e_{\xi}$ est convexe et donc $E$ aussi. Plus précisément, comme la base propre de $d f$ est 
orthogonale pour $D^{2} \eta$ et propre pour $d M_{\xi}$, il vient

$$
D_{M_{\xi}(s)}^{2} e_{\xi}\left(r_{j}(s), r_{k}(s)\right)=\frac{D^{2} \eta\left(r_{j}(s), r_{j}(s)\right)}{\rho_{j}(\xi, s)} \delta_{j}^{k} .
$$

Si $D^{2} \eta>0$ on a donc aussi $D^{2} e_{\xi}>0$. Dorénavant nous noterons

$$
\alpha_{j}(\xi)=\inf _{s \in K} \rho_{j}(\xi, s), \quad \beta_{j}(\xi)=\sup _{s \in K} \rho_{j}(\xi, s) .
$$

Par continuité, nous avons $0<\alpha_{j}(\xi) \leqslant \beta_{j}(\xi)<+\infty$.

Faisons maintenant l'hypothèse naturelle

H6 Le système (1) admet une entropie $\hat{\eta}$ qui vérifie $D^{2} \hat{\eta}>0$ sur $K$.

Nous pouvons ainsi normaliser les $r_{j}$ par $D^{2} \hat{\eta}\left(r_{j}, r_{j}\right) \equiv 1$.

On note alors $\hat{E}, \hat{F}, \hat{e}_{\xi}$ les fonctions correspondantes. Pour une solution $z^{\tau}$ de (12), nous avons

$$
\partial_{t} \hat{E}\left[z^{\tau}\right]+\partial_{x} \hat{F}\left[z^{\tau}\right]=\frac{1}{\tau} \int_{X} d \hat{e}_{\xi}\left(z^{\tau}\right) \cdot\left(M_{\xi}\left(u^{\tau}\right)-z^{\tau}(\xi)\right) d \mu(\xi) .
$$

Comme $d \hat{e}_{\xi}\left(M_{\xi}\left(u^{\tau}\right)\right)=d \eta\left(u^{\tau}\right)$ est indépendant de $\xi$, et puisque

$$
\int_{X}\left(M_{\xi}\left(u^{\tau}\right)-z^{\tau}(\xi)\right) d \mu(\xi)=0
$$

on peut réécrire (13) sous la forme

$$
\begin{aligned}
\partial_{t} \hat{E} & {\left[z^{\tau}\right]+\partial_{x} \hat{F}\left[z^{\tau}\right] } \\
& =\frac{1}{\tau} \int_{X}\left(d \hat{e}_{\xi}\left(z^{\tau}\right)-d \hat{e}_{\xi}\left(M_{\xi}\left(u^{\tau}\right)\right)\right) \cdot\left(M_{\xi}\left(u^{\tau}\right)-z^{\tau}(\xi)\right) d \mu(\xi) .
\end{aligned}
$$

Le second membre de (14) est négatif, puisque $d \hat{e}_{\xi}$ est monotone (convexité de $\hat{e}_{\xi}$ ), mais on peut le majorer de manière plus précise par

$$
-\frac{1}{\tau} \int_{X} \sum_{j=1}^{n} \alpha_{j}(\xi) d_{j}^{\xi}\left(z^{\tau}(\xi), M_{\xi}\left(u^{\tau}\right)\right)^{2} d \mu(\xi),
$$

où

$$
d_{j}^{\xi}(a, b):=\left(\int_{0}^{1}\left|l_{j} \circ M_{\xi}^{-1}(s a+(1-s) b) \cdot(b-a)\right|^{2} d s\right)^{1 / 2}
$$


est une semi-distance $\left(d^{\xi}(a, b):=\sum_{j} d_{j}^{\xi}(a, b)\right.$ est une distance équivalente à la distance usuelle sur $K)$. En intégrant (14) sur $\mathbb{R} \times(0, T)$, on obtient une borne indépendante de $\tau$ pour l'expression positive

$$
\frac{1}{\tau} \int_{X} \sum_{j=1}^{n} \alpha_{j}(\xi) d_{j}^{\xi}\left(z^{\tau}(\xi), M_{\xi}\left(u^{\tau}\right)\right)^{2} d \mu(\xi) .
$$

Ce calcul montre que, lorsque $\tau$ tend vers zéro, $z^{\tau}-\mathcal{M}\left[u^{\tau}\right]$ tend vers zéro dans un certain espace de Hilbert, en étant de l'ordre de $\tau^{1 / 2}$. L'approximation est donc consistante.

Compacité par compensation. - Etant donné un autre couple entropieflux $(\eta, q)$, étendu à $D$ en $(E, F)$, le même calcul fournit la majoration

$$
\left|\partial_{t} E\left[z^{\tau}\right]+\partial_{x} F\left[z^{\tau}\right]\right| \leqslant \frac{1}{\tau} \int_{X} \sum_{j=1}^{n} \beta_{j}(\xi) d_{j}^{\xi}\left(z^{\tau}(\xi), M_{\xi}\left(u^{\tau}\right)\right)^{2} d \mu(\xi) .
$$

La quantité $\left|\partial_{t} E\left[z^{\tau}\right]+\partial_{x} F\left[z^{\tau}\right]\right|$ reste donc bornée dans $L^{1}(\mathbb{R} \times(0, T))$, sous l'hypothèse supplémentaire d'uniformité :

H7 Les rapports $\beta_{j}(\xi) / \alpha_{j}(\xi)$ sont bornés sur $X$.

Cette condition est trivialement satisfaite si $X$ est fini. Elle l'est aussi si le choix des Maxwelliennes $M_{\xi}$ n'est pas "excentrique".

Montrons maintenant que la dissipation d'entropie $\partial_{t} \eta\left(u^{\tau}\right)+\partial_{x} q\left(u^{\tau}\right)$ reste dans un compact de $H_{l o c}^{-1}$. C'est le même calcul que pour (2) : cette dissipation est dans un borné de $W^{-1, \infty}$ (vu la stabilité $L^{\infty}$ ) et c'est la somme de $\partial_{t} E\left[z^{\tau}\right]+\partial_{x} F\left[z^{\tau}\right]$, qui est borné dans $L^{1}$, et de

$$
\partial_{t}\left(\eta\left(u^{\tau}\right)-E\left[z^{\tau}\right]\right)+\partial_{x}\left(q\left(u^{\tau}\right)-F\left[z^{\tau}\right]\right) .
$$

On montre à nouveau que chacune de ces deux dérivées est une suite qui tend vers zéro dans $H^{-1}$ fort. Par exemple,

$$
\begin{aligned}
& \eta\left(u^{\tau}\right)-E\left[z^{\tau}\right] \\
& \quad=E\left[\mathcal{M}\left[u^{\tau}\right]\right]-E\left[z^{\tau}\right] \\
& \quad=\int_{X}\left\{e_{\xi}\left(M_{\xi}\left(u^{\tau}\right)\right)-e_{\xi}\left(z^{\tau}\right)-d e_{\xi}\left(M_{\xi}\left(u^{\tau}\right)\right)\right\} \cdot\left(M_{\xi}\left(u^{\tau}\right)-z^{\tau}\right) d \mu(\xi),
\end{aligned}
$$

de sorte que $\eta\left(u^{\tau}\right)-E\left[z^{\tau}\right]$ est un $\mathcal{O}\left(\tau^{1 / 2}\right)$ dans $L^{2}$, en raison de l'estimation (15). Le lemme de Murat permet donc de conclure : $\partial_{t} \eta\left(u^{\tau}\right)+\partial_{x} q\left(u^{\tau}\right)$ reste dans un compact de $H_{l o c}^{-1}$. 
La suite $u^{\tau}$ étant bornée dans $L^{\infty}$ (elle est à valeurs dans $K$ ), on peut lui associer au moins une mesure de Young et celle-ci satisfait, d'après ce qui précède, l'équation de Tartar. Sous la même hypothèse qu'aux paragraphes précédents, on en déduit que cette mesure de Young est, pour presque tout $(x, t)$, une masse de Dirac : une suite extraite de $u^{\tau}$ converge presque partout. Notons $u$ sa limite. Si $\eta$ est convexe, alors $\partial_{t} \eta\left(u^{\tau}\right)+\partial_{x} q\left(u^{\tau}\right)$ est la somme d'une expression négative (à savoir $\left.\partial_{t} E\left[z^{\tau}\right]+\partial_{x} F\left[z^{\tau}\right]\right)$ et d'une distribution qui tend vers zéro dans $H^{-1}$, comme on l'a vu plus haut. Passant à la limite, on obtient donc l'inégalité

$$
\partial_{t} \eta(u)+\partial_{x} q(u) \leqslant 0,
$$

pour toute entropie convexe de (1), son flux étant noté $q$. Ainsi $u$ est solution entropique du problème de Cauchy pour (1).

Le résultat obtenu est le suivant :

THÉORÈme 5.2. - On fait les hypothèses $\mathbf{H 1}$ à $\mathbf{H 7}$ ci-dessus et on suppose aussi que l'équation de Tartar n'a pas d'autre solution que les masses de Dirac.

Soit $z_{0}: \mathbb{R} \rightarrow D$ une fonction mesurable. Alors le problème de Cauchy pour (12) admet une et une seule solution à valeurs dans $D$, définie sur $\mathbb{R} \times \mathbb{R}^{+}$, notée $z^{\tau}$. La suite $u^{\tau}$ est relativement compacte dans $L_{\text {loc }}^{p}\left(\mathbb{R} \times \mathbb{R}^{+}\right)$, pour tout $p<\infty$, et toute valeur d'adhérence est une solution entropique de (1), pour la donnée initiale

$$
u_{0}:=\int_{X} z_{0}(\xi) d \mu(\xi) .
$$

\section{RÉFÉRENCES}

[1] Brenier Y., Corrias L., Natalini R., A relaxation approximation to a moment hierarchy of conservation laws with kinetic formulation, Quaderno IAC 23 (1997).

[2] Chen G.Q., Liu T.P., Zero relaxation and dissipation limits for hyperbolic conservation laws, Comm. Pure Appl. Math. 46 (1994) 787-830.

[3] Chen G.Q., Levermore C.D., Liu T.P., Hyperbolic conservation laws with stiff relaxation terms and entropy, Comm. Pure Appl. Math. 45 (1993) 755-781.

[4] Chuey K.N., Conley C.C., Smoller J.K., Positively invariant regions of nonlinear diffusion equations, Indiana Univ. Math. J. 26 (1977) 373-392.

[5] Collet J.-F., Rascle M., Convergence of the relaxation approximation to a scalar nonlinear hyperbolic equation arising in chromatography, Z. Angew. Math. Phys. 47 (1996) 400-409. 
[6] DiPerna R.J., Convergence of approximate solutions to conservation laws, Arch. Rat. Mech. Anal. 82 (1983) 27-70.

[7] Heibig A., Existence and uniqueness of solutions for some hyperbolic systems of conservation laws, Arch. Rat. Mech. Anal. 126 (1994) 79-101.

[8] Hoff D., Invariant regions for systems of conservation laws, Trans. Amer. Math. Soc. 289 (1985) 591-610.

[9] Jin S., A convex entropy for a hyperbolic system with relaxation, J. Differential Equations 127 (1996) 95-107.

[10] Jin S., Xin Z., The relaxation schemes for systems of conservation laws in arbitrary space dimensions, Comm. Pure Appl. Math. 48 (1995) 235-277.

[11] Lattanzio C., Serre D., Stability and convergence of relaxation schemes towards systems of conservation laws, Soumis.

[12] Liu T.-P., Hyperbolic conservation laws with relaxation, Comm. Math. Phys. 108 (1987) 153-175.

[13] Murat F., L'injection du cône positif de $H^{-1}$ dans $W^{-1, q}$ est compacte pour tout $q<2$, J. Math. Pures et Appl. 60 (1981) 309-322.

[14] Natalini R., Convergence to equilibrium for the relaxation approximation of conservation laws, Comm. Pure Appl. Math. 49 (1996) 795-823.

[15] Natalini R., Recent results on hyperbolic relaxation problems, in: Analysis of Systems of Conervation Laws, Aachen, 1997, Chapman \& Hall/CRC Monogr. Surv. Pure Appl. Math., Vol. 99, Chapman \& Hall/CRC, Boca Raton, FL, 1999, pp. 128-197.

[16] Natalini R., A discrete kinetic approximation of entropy solutions to multidimensional scalar conservation laws, J. Differential Equations 148 (2) (1998) 292-317.

[17] Schochet S., The instant-response limit in Whitham's nonlinear traffic-flow model: uniform well-posedness, Asymptotic Anal. 1 (1988) 263-282.

[18] Serre D., Systèmes de Lois de Conservation, Diderot, Paris, 1996.

[19] Serre D., Shearer J., Convergence with physical viscosity for nonlinear elasticity, Preprint éternel, Lyon, 1993.

[20] Shearer J., Global existence and compactness in $L^{p}$ for the quasi-linear wave equation, Comm. Partial Differential Equations 19 (1994) 1829-1877.

[21] Tartar L., Compensated compactness and applications to partial differential equations, in: Knops R.J. (Ed.), Nonlinear Analysis and Mechanics, Heriot-Watt Symposium, Research Notes in Math., Vol. 39, Pitman, Londres, 1979, pp. 136-192.

[22] Tveito A., Winther R., On the rate of convergence to equilibrium for a system of conservation laws with a relaxation term, SIAM J. Math. Anal. 28 (1997) 136161.

[23] Tzavaras A., Materials with internal variables and relaxation to conservation laws, Preprint, Madison, 1998.

[24] Whitham J., Linear and Non-Linear Waves, Wiley, New York, 1974. 